\title{
Optical Axis Perturbation Analysis for the Unit-Magnification Multipass System
}

\author{
Yin Guo, Liqun Sun, and Zheng Yang \\ State Key Laboratory of Precision Measurement Technology and Instrument, Department of Precision Instruments, Tsinghua University, \\ Beijing 100084, China
}

Correspondence should be addressed to Liqun Sun; sunlq@mail.tsinghua.edu.cn

Received 13 January 2017; Accepted 26 January 2017; Published 20 February 2017

Academic Editor: Ivan D. Rukhlenko

Copyright (C) 2017 Yin Guo et al. This is an open access article distributed under the Creative Commons Attribution License, which permits unrestricted use, distribution, and reproduction in any medium, provided the original work is properly cited.

The optical axis sensitivity for the unit-magnification multipass system (UMS) is presented by using a general misaligned optical element transfer model. The generalized sensitivity factors SD1, SD2, ST1, and ST2 influenced by both the axial and angular misalignments of the objective mirrors in a UMS have been calculated for the first time. The Bernstein-Herzberg White Cells are used as an example, and their alignment tolerance and stability properties are found when their configurations change. The analysis in this paper is helpful for the design of other kinds of multipass gas cells (MGC) with high robustness and avoiding the violent vibration of the optical axis when the misalignment of each mirror is controlled within the tolerance range. Among the five possible perturbations sources, the misaligned factors $\left(\delta_{i x}, \delta_{i y}, \theta_{i x}\right)$ have more effects on the output beam's position and the perturbed sources from $\left(\delta_{i x}, \theta_{i x}\right)$ and $\left(\delta_{i y}, \theta_{i y}\right)$ have more impacts on the output beam's slope referred to as $x$-axis and $y$-axis, respectively. Higher reflection times mean smaller tolerance range. The results benefit the multipass cell design and the precise alignment of the mirrors within the cell with the purpose of long-term stability in measurements.

\section{Introduction}

Unit-magnification multipass system (UMS) [1] has been widely used in the absorption spectroscopy instrument for decades in the field of industrial and environmental science [2]. White cell [3], Herriott cell [4], circular cell [5], and Chernin-type systems, that is, the multipass matrix system (MMS), are the most general categories of the multipass system to obtain very long optical absorption path length in compact space to enhance the detection sensitivity. The white cell and MMS are widely known for their relatively large numerical aperture and large volume [1]. This kind of cell is commonly applied to broadband absorption spectroscopy to retrieve multiple species, such as ultraviolet differential optical absorption spectroscopy (UV-DOAS) and Fourier transform infrared spectroscopy (FTIR). On the other hand, Herriott-type cell and circular cell are commonly applied to the laser-based absorption spectroscopy and can provide very long path length in relatively small volume. These cells are usually applied to harsh in situ gas sensing environment, and highly stable conjugated alignment conditions of optical elements are required for a high throughput signal. No matter what kind of cell is used, optical system's stability, namely, optical axis stability properties, is an important issue when used in the long term. Vibration-induced misalignment of the mirror in position will make the output signal intensity reduce. In that case, realignment of the mirror or calibration of the system is required. Tolerance data of a given UMS is calculated in this paper for the optomechanical engineers to ensure the long-term stability under harsh environment. Meanwhile, the tolerance of the reflective optics in the UMS is also investigated considering the different configuration parameters.

The UMS is noncoaxial, noncoplanar, and partly asymmetric which can be regarded as the multipass 3D optical system [1]. It consists of two blocks of spherical mirrors with identical curvature radii $R$, objective mirrors $\left(M_{i}\right)$ on one block, and the field mirrors $\left(F_{i}\right)$ on the other side of block face-to-face with an interval, approximately $R$. The entrance aperture is placed on one side of the field mirror, and output window lies on the other side of the field mirror. After travelling multiple passes in the UMS, the light 
source formed multiple images with almost the same size on the field mirror. Previous researchers focused on the astigmatism calculation and its correction strategy by using reversed astigmatism compensating methods $[1,6]$. It aims at obtaining less degraded beam when the beam traveled out from the output window, especially for the white cell series, that is, Bernstein-Herzberg White Cell (BHWC) $[7,8]$ and Pickett-Bradley White Cell (PBWC) [9]. Other various kinds of white cell are based on these two basic configurations to get enhanced spot pattern on the field mirror, that is, Chernintype cell $[10,11]$ (three-objective matrix multipass system and four-objective matrix multipass system). However, the analysis of the optical axis perturbation properties for a certain UMS has not been reported before. For a given UMS, its optical layout is determined by a given number of passes, spot pattern, input beam's numerical aperture, and diameter. The consideration of the system's alignment properties and optical axis stability should be carefully concerned when constructing the system and keep them in a robust condition for long-term measurements. In this case, an unstable or lack of tolerance analysis for the fabrication and alignment control to these multiple mirrors in the UMS would lead to some unexpected results, for example, temperature and pressure induced misalignment or the excess of alignment tolerance in the mechanical construction. In other words, the optimized choice of UMS configuration parameters may benefit the long-term measurements and avoid absorption path length variations induced by misalignment of the optical elements or signal obstruction. Moreover, the optical axis sensitivity analysis will also improve the alignment process.

Ray matrix method has been widely used to analyze optical axis perturbations in various 3D optical systems [12-15]. Sheng [12] used a matrix approach to determine the optical axis perturbation sensitivity of an out-of-plane stable ring resonator. Based on the augmented $5 \times 5$ ray matrix method, Chen et al. [13] presented general calculation method of nonplanar ring resonator's (Zero-Lock Laser Gyroscopes) beam position and proposed precise beam controlling way. Yuan and Long [14] obtained optical axis stable region in terms of axis decentration and tilt with augmented $5 \times 5$ matrix formulations. After that, they reanalyzed the sensitivity factors with the proposed merit indexes to characterize the nonplanar ring resonator with the same method within the defined coordinate system [15]. Therefore, by employing the augmented $5 \times 5$ ray matrix method, beam behavior in our multipass systems (also called UMS) can be modeled in the paraxial approximation domain (incident angle less than 5 degrees). In this paper, we develop a numerical method by incorporating $5 \times 5$ augmented ray matrix to analyze the optical axis sensitivity in the UMS. Any optics elements in the UMS have their possible perturbations, thus resulting in the error on the output beam in position and direction. The spherical reflection mirror's transfer matrix is derived based on a general model of the paraxial misaligned optical element. Considering the imaging rotation due to the changing of reflection plane and coordinate system in each reflection, the rotation matrix is used. The extent of the influence of the output beam is different depending on the choice of UMS's configuration parameters. By utilizing the

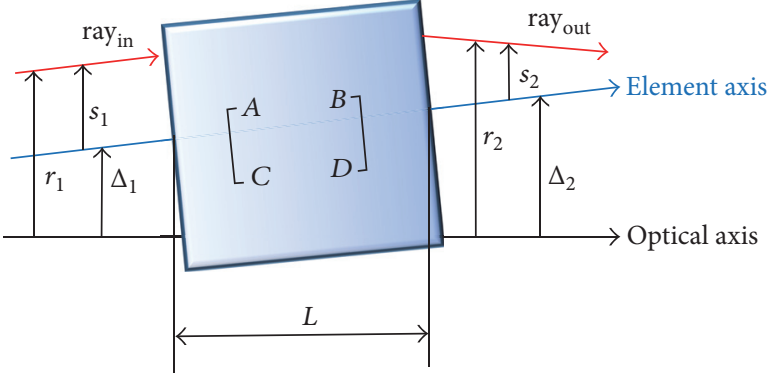

FIGURE 1: The general model of a paraxial misaligned optical element.

defined sensitivity factors influenced by both the axial and angular misalignments of the objective mirror in the UMS, the rule of optical axis perturbation is investigated in detail. According to the different initial optical layout configurations of the UMS, the numerical simulation presents the rule of optical axis sensitivity, and the primary perturbation sources are summarized. BHWC is used as an example to analyze its optical axis sensitivity. The method proposed can also be applied to other various multipass cavity cells and their preliminary design.

\section{General Analysis of a Misaligned Optical System}

The general ray matrix of an optical system with axial and angular misalignment can be modeled by using the following form [16]:

$$
\left(\begin{array}{c}
r_{o x} \\
r_{o x}^{\prime} \\
r_{o y} \\
r_{o y}^{\prime} \\
1
\end{array}\right)=\left(\begin{array}{ccccc}
A_{x} & B_{x} & 0 & 0 & E_{x} \\
C_{x} & D_{x} & 0 & 0 & F_{x} \\
0 & 0 & A_{y} & B_{y} & E_{y} \\
0 & 0 & C_{y} & D_{y} & F_{y} \\
0 & 0 & 0 & 0 & 1
\end{array}\right)\left(\begin{array}{c}
r_{i x} \\
r_{i x}^{\prime} \\
r_{i y} \\
r_{i y}^{\prime} \\
1
\end{array}\right),
$$

where $\left(r_{i x}, r_{i x}^{\prime}, r_{i y}, r_{i y}^{\prime}, 1\right)^{T}$ and $\left(r_{o x}, r_{o x}^{\prime}, r_{o y}, r_{o y}^{\prime}, 1\right)^{T}$ are the input and output ray matrix, respectively, referred to as the reference plane. $r_{i x}, r_{i y}, r_{o x}$, and $r_{o y}$ are the input ray and output ray height in the reference $x o z$ and yoz plane, respectively, and are called optical axis decentration. $r_{i x}^{\prime}, r_{i y}^{\prime}$, $r_{o x}^{\prime}$, and $r_{o y}^{\prime}$ are the angles (in radians) of the input ray and output ray slope in the reference $x o z$ and yoz plane, respectively, and are referred to as optical axis tilt. $A_{x}, B_{x}, C_{x}$, and $D_{x}$ are the standard ray matrix elements in the tangential plane (xoz plane). $A_{y}, B_{y}, C_{y}$, and $D_{y}$ are the standard ray matrix elements in the sagittal plane (yoz plane).

To solve the misaligned value for the transmission matrix analytically in our system (see (1)), we firstly construct a general model of a misaligned optical element [17], illustrated in Figure 1.

A misaligned optical element in the paraxial domain can be regarded as the case when the element axis does not coincide with the optical axis. Any optical elements have 
six possible degrees of freedom (three in axial and three in angular error) relative to the element axis. Optical axis can be defined arbitrarily to describe ray vector. Conveniently, we take ray's propagation in one tangential plane as an example as shown in Figure 1. Ray vector referred to the element axis, and the optical axis can be written as $\left[s_{i}, s_{i}^{\prime}\right]$ and $\left[r_{i}, r_{i}^{\prime}\right]$, respectively, where $s_{i}$ and $r_{i}$ denote the displacement in the reference plane; $s_{i}^{\prime}$ and $r_{i}^{\prime}$ represent the angle in radian in the reference plane. For the two particular axes illustrated in Figure 1, the element axis can be expressed relative to the optical axis by using a vector $\left[\Delta_{i} \Delta_{i}^{\prime}\right]$, where $\Delta_{i}$ is the height of axial vector and $\Delta_{i}^{\prime}$ is the slope in a radian of the axial vector in the reference plane ( $i=1$ denotes the objective spacing and $i=2$ denotes the imaging spacing). Considering the paraxial approximation when in condition of possible perturbation, we have the following relationships of the perturbed element axis between input and output plane:

$$
\Delta^{\prime}=\frac{\Delta_{2}-\Delta_{1}}{L} \text {. }
$$

Then, we introduce our misalignment vectors to describe our misaligned values $P_{1} \equiv\left[\begin{array}{c}\Delta_{1} \\ \Delta_{1}^{\prime}\end{array}\right]$ and $P_{2} \equiv\left[\begin{array}{c}\Delta_{2} \\ \Delta_{2}^{\prime}\end{array}\right]$, where $\Delta_{1}=$ $n_{1} \Delta^{\prime}, \Delta_{2}=n_{2} \Delta^{\prime}$, and $n_{1}$ and $n_{2}$ are the refractive indices of input and output space, respectively. The connection between the two misaligned vectors can be written as

$$
P_{2}=P_{\Delta} \cdot P_{1}
$$

where $P_{\Delta}=\left[\begin{array}{ll}1 & L / n_{1} \\ 0 & n_{2} / n_{1}\end{array}\right]$.

Moreover, ray vector referred to the optical axis and the element axis in the input and output ends can be described as

$$
\begin{gathered}
r_{\text {in }}=\left[\begin{array}{l}
r_{1} \\
r_{1}^{\prime}
\end{array}\right]=\left[\begin{array}{l}
s_{1} \\
s_{1}^{\prime}
\end{array}\right]+\left[\begin{array}{l}
\Delta_{1} \\
\Delta_{1}^{\prime}
\end{array}\right]=s_{\text {in }}+P_{1}, \\
r_{o}=\left[\begin{array}{l}
r_{2} \\
r_{2}^{\prime}
\end{array}\right]=\left[\begin{array}{l}
s_{2} \\
s_{2}^{\prime}
\end{array}\right]+\left[\begin{array}{l}
\Delta_{2} \\
\Delta_{2}^{\prime}
\end{array}\right]=s_{o}+P_{2} .
\end{gathered}
$$

Because the system transmission matrix related to the element axis can be regarded as nonmisaligned system, which follows the law of traditional $A B C D$ matrix measured on the input and output plane, we can connect $s_{\text {in }}$ and $s_{o}$ by using the following formula:

$$
\left[\begin{array}{l}
s_{2} \\
s_{2}^{\prime}
\end{array}\right]=\left[\begin{array}{ll}
A & B \\
C & D
\end{array}\right] \cdot\left[\begin{array}{l}
s_{1} \\
s_{1}^{\prime}
\end{array}\right] .
$$

Up to now, we can put (6), (4), and (3) into (5); we can easily get the output ray vector written as

$$
\begin{aligned}
r_{o} & =\left[\begin{array}{l}
r_{2} \\
r_{2}^{\prime}
\end{array}\right]=M \cdot s_{\text {in }}+M_{\Delta} \cdot P_{1} \\
& =M \cdot r_{\text {in }}+\left[M_{\Delta}-M\right] P_{1}=M \cdot r_{\text {in }}+E_{M},
\end{aligned}
$$

where the matrix $E_{M(2 \times 1)}$ is the error matrix induced by possible misalignment error; it is defined as

$$
\begin{gathered}
E_{M} \stackrel{\text { def }}{=}\left[M_{\Delta}-M\right] P_{1}=\left[\begin{array}{cc}
1-A & \frac{L}{n_{1}}-B \\
-C & \frac{n_{2}}{n_{1}}-D
\end{array}\right]\left[\begin{array}{l}
\Delta_{1} \\
\Delta_{1}^{\prime}
\end{array}\right] \\
\stackrel{\Delta_{1}^{\prime}=n_{1} \Delta^{\prime}}{=}\left[\begin{array}{cc}
1-A & L-n_{1} B \\
-C & n_{2}-n_{1} D
\end{array}\right]\left[\begin{array}{l}
\Delta_{1} \\
\Delta^{\prime}
\end{array}\right]=\left[\begin{array}{c}
E_{i} \\
F_{i}
\end{array}\right],
\end{gathered}
$$

where $i=x$ and $i=y$ represent the error matrix in tangential plane and sagittal plane, respectively.

We use these results for a general misaligned paraxial optical system in one tangential plane. Thus, we arrive at 3 $\times 3$ transmission matrix:

$$
\left[\begin{array}{c}
r_{o x} \\
r_{o x}^{\prime} \\
1
\end{array}\right]=\left[\begin{array}{ccc}
A_{x} & B_{x} & E_{x} \\
C_{x} & D_{x} & F_{x} \\
0 & 0 & 1
\end{array}\right]\left[\begin{array}{c}
r_{i x} \\
r_{i x}^{\prime} \\
1
\end{array}\right] .
$$

The matrix $E_{M(2 \times 1)}$ can be extended to get displacement error matrix $E_{x}$ and angle error matrix $F_{x}$ and thus we have solved $E_{x}$ and $F_{x}$ as follows:

$$
\begin{aligned}
& E_{x}=\left(1-A_{x}\right) \Delta_{1}+\left(L-n_{1} B_{x}\right) \Delta^{\prime}, \\
& F_{x}=-C_{x} \Delta_{1}+\left(n_{2}-n_{1} D_{x}\right) \Delta^{\prime} .
\end{aligned}
$$

According to the method described above, the specific values of $E_{x}, F_{x}, E_{y}$, and $F_{y}$ in (1) can be derived in given perturbation amount for an individual paraxial optical element.

Because all optical elements in the UMS are the spherical mirror, it is necessary to analyze the generalized transmission matrix of spherical mirror in case of all kinds of possible perturbation based on (10).

A spherical mirror $M_{i}\left(\operatorname{radii} R_{i}\right)$ has been chosen as an example to demonstrate the misaligned sources in Figure 2. The incident angle is $A_{i}\left(\right.$ smaller than $\left.5^{\circ}\right) ; \delta_{i x}, \delta_{i y}$, and $\delta_{i x}$ are its three kinds of axial displacements along the axes $T_{i x}, T_{i y}$, and $T_{i z}$, respectively. $\theta_{i z}, \theta_{i x}$, and $\theta_{i y}$ are three types of angular misalignments around the rotational axes of $R_{i x}, R_{i y}$, and $R_{i z}$, respectively. The term $\theta_{i z}$ can be ignored due to the symmetry of the spherical mirror.

The terms $\theta_{i x}, \theta_{i y}, \delta_{i x}, \delta_{i y}$, and $\delta_{i z}$ are used to describe five kinds of possible perturbation sources for a spherical mirror. In this paper, $\theta_{i x}$ and $\theta_{i y}$ are angular misalignments; $\delta_{i x}, \delta_{i y}$, and $\delta_{i z}$ are called axial displacements or axial misalignments.

In [16], Yuan et al. have discussed and derived the generalized ray matrix for a spherical mirror reflection in a square ring resonator. They proposed generalized spherical mirror reflection-transmission matrix based on the geometrical method. In this paper, we derived the generalized spherical mirror reflection matrix based on a general misaligned optical element model to deduce the error matrix 


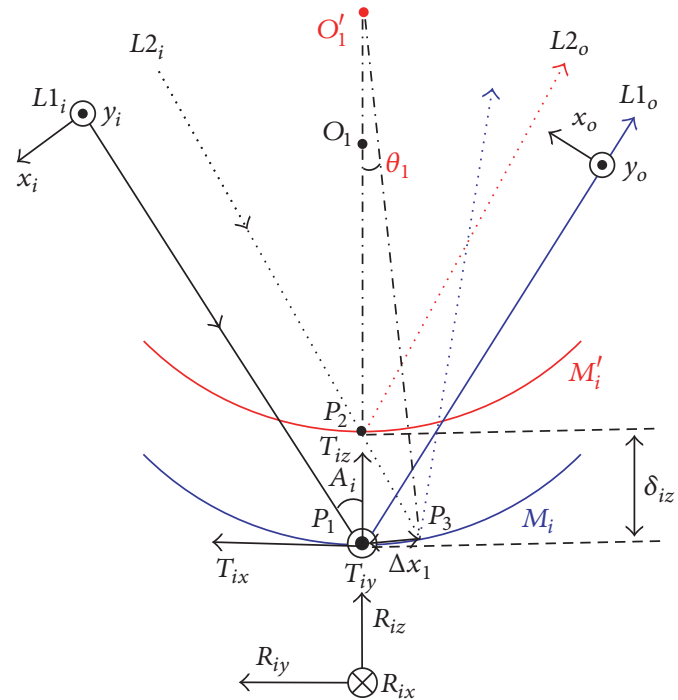

(a)

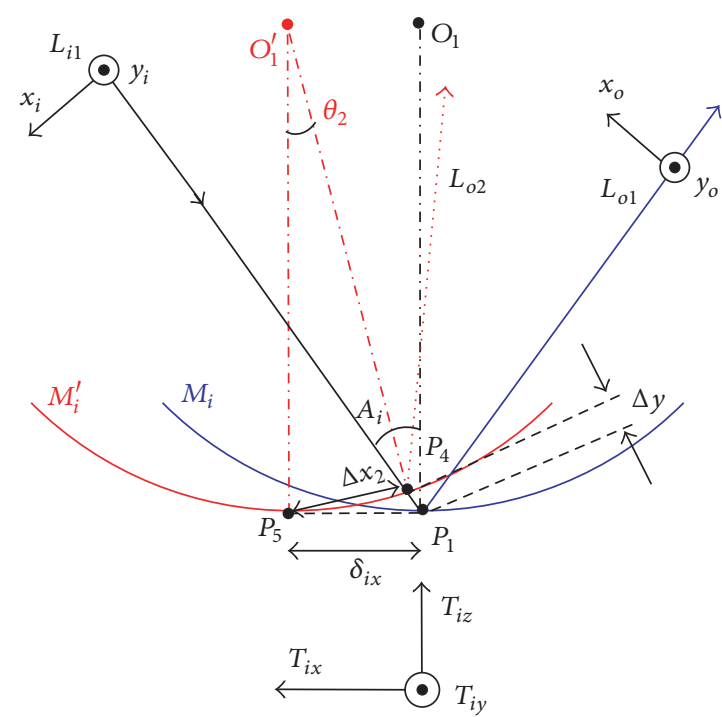

(b)

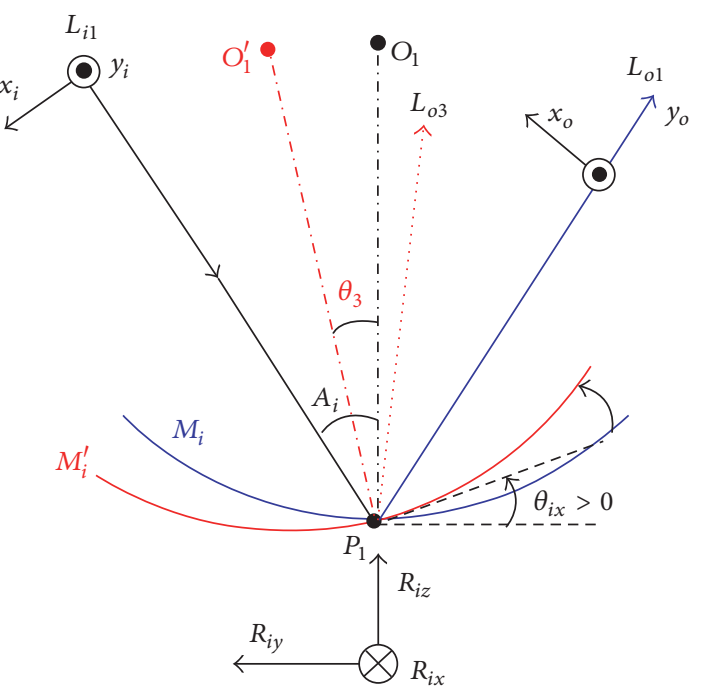

(d)

FIGURE 2: Axial and radial misalignment analysis of a spherical mirror $M_{i}$. (a) Axial displacement $\delta_{i z}$ of $M_{i}$ along the translational axis $T_{z}$. (b) Axial displacement $\delta_{i x}$ of $M_{i}$ along the translational axis $T_{x}$. (c) Definition of a mirror's angular misalignments $\theta_{i x}$ and (d) angular misalignment of the spherical mirror $M_{i}$ around rotational axis $R_{i x} . A_{i}$ is the incident angle, $M_{i}$, the blue solid arc, is the mirror's initial position, $M_{i}^{\prime}$ and $M_{i}^{\prime \prime}$ in the red solid arc in (a), (b), (c), and (d) are the position of the mirror after possible axial or angular perturbations. $P_{1}, P_{2}, P_{3}, P_{4}$, and $P_{5}$ are the reflecting points. $P_{0}$ is a point on ray $L_{i 1} ; O_{1}$ and $O_{1}^{\prime}$ are the curvature centers of $M_{i}$ and $M_{i}^{\prime} . T_{i x}, T_{i y}$, and $T_{i z}$ are three translational axes (right hand); $R_{i x}, R_{i y}$, and $R_{i z}$ are three rotational axes (right hand); $L 1_{i}$ and $L 2_{i}$ are two parallel incident rays; $L_{i 1}$ is an incident ray; $L_{o 1}$ and $L_{o 2}$ are two reflection rays; $x_{i}-y_{i}$ are the coordinate axes (left hand) of the incident ray; $x_{o}-y_{o}$ are the coordinate axes of the reflected ray; $\theta_{1}$ is the angle between line $O_{1}^{\prime} P_{1}$ and line $O_{1}^{\prime} P_{3} ; \theta_{2}$ is the angle between line $O_{1}^{\prime} P_{4}$ and line $O_{1}^{\prime} P_{5} ; \theta_{3}$ is the angle between line $O_{1}^{\prime} P_{1}$ and line $O_{1} P_{1} ; \Delta x_{1}$ is the distance between $P_{1}$ and $P_{3} ; \Delta x_{2}$ is the distance between $P_{4}$ and $P_{5} ; \Delta y$ is the distance between $P_{1}$ and $P_{4}$.

$\left[\begin{array}{llll}E_{x} & F_{x} & E_{y} & F_{y}\end{array}\right]^{T}$ in (1). It can be rewritten as the following equations:

$$
\begin{aligned}
& E_{x}=\left(1-A_{x}\right) \Delta_{1 x}+\left(L-n_{1} B_{x}\right) \Delta_{x}^{\prime}, \\
& F_{x}=-C_{x} \Delta_{1 x}+\left(n_{2}-n_{1} D_{x}\right) \Delta_{x}^{\prime}, \\
& E_{y}=\left(1-A_{y}\right) \Delta_{1 y}+\left(L-n_{1} B_{y}\right) \Delta_{y}^{\prime}, \\
& F_{y}=-C_{y} \Delta_{1 y}+\left(n_{2}-n_{1} D_{y}\right) \Delta_{y}^{\prime} .
\end{aligned}
$$

As is widely known for the reflection system, $n_{1}=-n_{2}=$ 1. For a spherical mirror, its ray transfer matrix (radius $R_{i}$ with incident angle $A_{i}$ ) can be written as

$$
\left[\begin{array}{cccc}
A_{x} & B_{x} & 0 & 0 \\
C_{x} & D_{x} & 0 & 0 \\
0 & 0 & A_{y} & B_{y} \\
0 & 0 & C_{y} & D_{y}
\end{array}\right]=\left[\begin{array}{cccc}
1 & 0 & 0 & 0 \\
-f_{m}^{-1} & 1 & 0 & 0 \\
0 & 0 & 1 & 0 \\
0 & 0 & -f_{s}^{-1} & 1
\end{array}\right],
$$


where $f_{m}=-R_{i} \cos \left(A_{i}\right) / 2$ is the focal length in meridional plane $(x-z$ plane $)$ and $f_{s}=-R_{i} / 2 \cos \left(A_{i}\right)$ is the focal length in the sagittal plane ( $y-z$ plane). Therefore (11) (14) can also be simplified as the following expression in the case of spherical reflection mirror:

$$
\begin{aligned}
& E_{x}=\Delta_{2 x}-\Delta_{1 x}, \\
& F_{x}=\frac{2}{R_{i} \cos \left(A_{i}\right)} \Delta_{1 x}-2 \times \Delta_{x}^{\prime}, \\
& E_{y}=\Delta_{2 y}-\Delta_{1 y}, \\
& F_{y}=\frac{2 \cos \left(A_{i}\right)}{R_{i}} \Delta_{1 y}-2 \Delta_{y}^{\prime} .
\end{aligned}
$$

To solve the terms $E_{x}, F_{x}, E_{y}$, and $F_{y}$, Figure 2 illustrates the diagram of optical axis movement. As shown in Figure 2(a), the axial displacement $\delta_{i z}$ is analyzed firstly, when $A_{i} \neq 0$ (the common case for the UMS). To discuss this effect in detail, two parallel incident rays $L 1_{i}$ and $L 2_{i}$ are chosen, for example. $L 1_{o}$ is the reflection ray at the point $P_{1}$ on $M_{i} . L 2_{o}$ is the reflection ray of $L 2_{i}$ via the reflection at the point $P_{2}$ on $M_{i}^{\prime}$. In the case of the displacement misalignment along $T_{i z}$ axis with $\delta_{i z}$ for the mirror $M_{i}$, the ray $L 2_{i}$ and $L 2_{o}$ is the perturbed incident and output compared with the incident ray $L 1_{i}$ and output ray $L 1_{0}$ before the mirror's perturbations. In that case, the reflecting point $P_{2}$ (a point on the element axis) relative to its optical axis before reflection and after reflection is expressed as $\Delta_{1 x}=-\delta_{i z} \sin \left(A_{i}\right)$ and $\Delta_{2 x}=$ $\delta_{i z} \sin \left(A_{i}\right)$, respectively. We put them into (16) and obtain $E_{x}=2 \delta_{i z} \sin \left(A_{i}\right)$. There is no change in slope between element axes in the input and output ends of the spherical mirror. We have $\Delta_{x}^{\prime}=0$ and then we can obtain $F_{x}=$ $-2 \delta_{i z} \tan \left(A_{i}\right) / R_{i}$ according to (17). Similarly, in the sagittal plane, $\Delta_{2 y}=0, \Delta_{1 y}=0$, and $\Delta_{y}^{\prime}=0$ as shown in Figure 2(a). So $E_{y}=F_{y}=0$ in this case. Therefore, if the axial displacement along the axis $T_{i z}$ is $\delta_{i z}$, the ray matrix elements $E_{x}$ and $F_{x}$ should be modified into $2 \delta_{i z} \cdot \sin \left(A_{i}\right)$ and $-2 \delta_{i z} \tan \left(A_{i}\right) / R$, respectively.

Next, the spherical mirror's other two axial displacements $\delta_{i x}$ and $\delta_{i y}$ need to be considered too. As illustrated in Figure $2(\mathrm{~b}), \delta_{i x}$ is chosen as an example for analysis. The line $O_{1}^{\prime} P_{4}$ is the element axis, the coordinates of point $P_{4}$ (a point at the element axis) referred to as the optical axis in the input ends and output ends are $\Delta_{1 x}=0$ and $\Delta_{2 x}=\Delta y \times \sin \left(2 A_{i}\right)$, respectively. Because $\Delta y \approx \Delta x_{2} \cdot \sin \left(\theta_{2}\right)$, for approximation, $\sin \left(\theta_{2}\right) \approx \theta_{2} \approx \Delta x_{2} / R_{i}$ and $\Delta x_{2} \approx \delta_{i x}$. Then the error matrix in this case becomes $E_{x}=\left(\delta_{i x}^{2} / R_{i}\right) \times \sin \left(2 A_{i}\right)$. This term can be ignored in this paper due to $\delta_{i x} \ll R_{i}$. The changing slope of the element axis between the input ends and the output is $\Delta_{x}^{\prime}$ relative to the optical axis. In our case, element axis from input ends referred to as the optical axis is the angle between the line $O_{1} P_{1}$ and the line along the incident ray $L_{i 1}$, while the element axis from the output ends referred to as the optical axis is the angle between the line $O_{1}^{\prime} P_{4}$ and the line along the incident ray $L_{i 1}$. Therefore $\Delta_{x}^{\prime}$ should be the angle change between line $O_{1} P_{1}$ and $O_{1}^{\prime} P_{4}$, namely, two element axes angles (initial element axis and perturbed element axis), $\theta_{2}$. As shown in Figure $2(\mathrm{~b}), \Delta_{x}^{\prime}=-\theta_{2}=-\delta_{i x} / R_{i}$. The error matrix $F_{x}=\left(2 / R_{i} \cos \left(A_{i}\right)\right) \Delta_{1 x}-2 \times \Delta_{x}^{\prime}=-2 \times\left(-\theta_{2}\right)=$ $2 \delta_{i x} / R_{i}$. Because $\delta_{i x}$ makes no contributions to $\Delta_{2 y}, \Delta_{1 y}$, and $\Delta_{y}^{\prime}, E_{y}$ and $F_{y}$ will be zero in this case. Similarly, influenced by $\delta_{i y}$ error matrix of $E_{y}=\left(\delta_{i y}^{2} / R_{i}\right) \times \sin \left(2 A_{i}\right)$, this term can also be ignored due to $\delta_{i y} \ll R_{i}$ and $F_{y}=\left(2 \cos \left(A_{i}\right) / R_{i}\right) \times$ $\Delta_{1 x}-2 \times \Delta_{x}^{\prime}=-2 \times\left(-\theta_{2}\right)=2 \delta_{i y} / R_{i}$ based on the same law. In summary, when the spherical mirror is misaligned by axial displacements $\delta_{i x}$ and $\delta_{i y}$, its ray matrix elements $F_{x}$ and $F_{y}$ should be modified into $2 \delta_{i x} / R_{i}$ and $2 \delta_{i y} / R_{i}$, respectively.

The angular misalignment $\theta_{i x}$ is chosen as an example to show the influence of angular perturbations (the analysis of angular misalignment $\theta_{i y}$ is similar; $\theta_{i z}$ can be ignored due to the symmetry shape of the spherical mirror). Figure 2(c) shows the definition of $\theta_{i x}$. We look at the mirror $M_{i}$ behind the rotation axis $R_{i x}$; when the mirror $M_{i}$ rotates clockwise on its rotation axis $R_{i x}$, the sign of misalignment angle of $\theta_{i x}$ is negative. When it rotates counterclockwise on its rotation axis $R_{i x}$, the sign of misalignment angle of $\theta_{i x}$ is positive. In Figure $2(\mathrm{~d})$, because the reflecting point $P_{1}$ on the element axis experiences no change in displacement referred to as the optical axis, we get the term $\Delta_{1 x}=\Delta_{2 x}=\Delta_{1 y}=\Delta_{2 y}=0$.

And there is no change in slope angle for element axis in $y z$ plane; we have $\Delta_{y}^{\prime}=0$. Element axis has slope variation between the input ends and the output ends referred to as the optical axis, from $\angle P_{0} P_{1} O_{1}$ to $\angle P_{0} P_{1} O_{1}^{\prime}$, so the term $\Delta_{x}^{\prime}=$ $-\theta_{3}=-\theta_{i x}$. According to (16) (19), the standard ray matrix element $F_{x}$ in (1) should be modified into $2 \theta_{i x}$ and $F_{y}$ should be modified into $2 \theta_{i y}$ with the consideration of the mirror's angular misalignment $\theta_{i y}$.

In summary, a generalized ray matrix $M\left(R_{i}, A_{i}\right)$ for a spherical mirror $M_{i}$ with all possible kinds of perturbation sources including $\theta_{i x}, \theta_{i y}, \delta_{i x}, \delta_{i y}$, and $\delta_{i z}$ can be written as

$$
M\left(R_{i}, A_{i}\right)=\left[\begin{array}{ccccc}
1 & 0 & 0 & 0 & 2 \delta_{i z} \cdot \sin \left(A_{i}\right) \\
-\frac{2}{R_{i} \cdot \cos \left(A_{i}\right)} & 1 & 0 & 0 & \frac{-2 \delta_{i z} \cdot \tan \left(A_{i}\right)}{R_{i}}+2\left(\theta_{i x}+\frac{\delta_{i x}}{R_{i}}\right) \\
0 & 0 & 1 & 0 & 0 \\
0 & 0-\frac{2 \times \cos \left(A_{i}\right)}{R_{i}} & 1 & 2\left(\theta_{i y}+\frac{\delta_{i y}}{R_{i}}\right) \\
1 & 0 & 0 & 0 &
\end{array}\right] .
$$




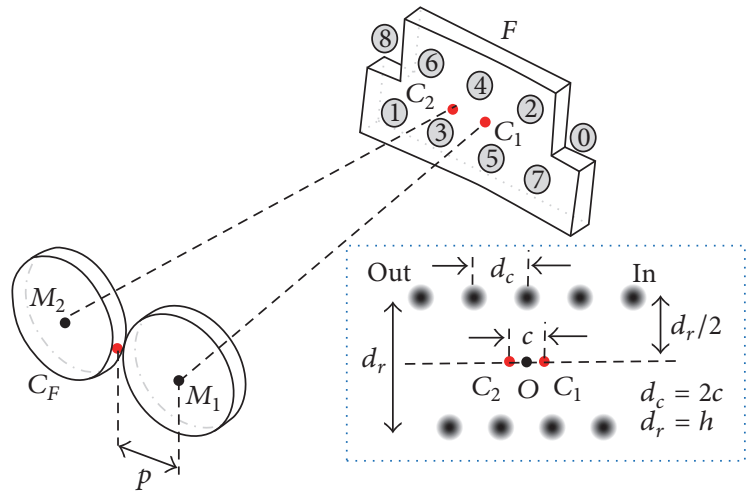

FIgURE 3: Schematic diagram of BHWC. Two objective mirrors $M_{1}$ and $M_{2}$ lay on one side and a field mirror $F$ locates on the other side with an interval close to $R$. All mirrors have identical radii of curvature $R . C_{i}(i=1,2, F)$ is the curvature center of each mirror. Two-row images are formed on the field mirror with the column spacing $d_{c}=2 c$ and row spacing $d_{r}=h$, where $c$ is the distance of curvature center of $M_{1}$ and $M_{2} . p$ is the center shift of the input beam which represents the center point on the mirror $M_{1}$.

Equation (20) is the same as Yuan et al. proposed [16], but it is explained and derived in a new way based on the modeling of a general misaligned optical element's ray matrix (shown in Figure 1). The misaligned matrix can be understood with the clear meaning of each symbol in definitions. The derivation process of Yuan et al. was based on the geometrical compensating method. These two methods can be mutually proven in different ways. Furthermore, the general misaligned optical transfer matrix model can be easily applied to other various optics elements in different kinds of the UMS.

\section{Analysis of the BHWC}

BHWC $[7,8]$ is one of the traditional and widely known optical schemes in the UMS. Another commonly used optical layout is the PBWC [9]. Other types of complicated multipass gas cell are the multiple usages of BHWC or PBWC to increase the number of reflection times when incorporated with different reentrant way. In this paper, we just discuss the BHWC in detail as an example.

As shown in Figure 3, we can use a parameter set ( $n$, $\left.R, d_{c}, d_{r}\right)$ to establish a UMS. $N$ is the total reflection times of the multipass cell. As for BHWC, $N=4 n(n=1,2,3, \ldots)$, where $n$ is the number of spots for the bottom row on the field mirror; for example, in Figure 3, $n=4$.

As is well known, the UMS are noncoaxial, noncoplanar, and partly asymmetric because the meridional planes of each reflection are not coplane. The optical transmission matrix can be modeled as several cascades misaligned optical elements. An individual reflection on a spherical mirror with an off-axis incident angle $A_{i}$ can be considered as a transmission through a thin astigmatic lens with its optical axis in the direction of a chief ray. The transfer matrix for the $j$ th reflection $M_{j}$ can be written as [18]

$$
M_{j}=R\left(\varphi_{j}\right) M\left(R, A_{j}\right) T\left(L_{j}\right),
$$

where $M\left(R, A_{j}\right)$ is the generalized spherical mirror's reflection matrix (described by $(20)$ ). $T\left(L_{j}\right)$ is $5 \times 5$ ray transfer matrix for free space propagation in a homogeneous medium and $R\left(\varphi_{j}\right)$ is the coordinate rotation matrix when considering the change of each reflective meridional plane, where $\varphi_{j}$ is the meridional plane rotation angle relative to the last meridional plane. $A_{j}$ is the incident angle of the $j$ th reflection on a spherical mirror. $T\left(L_{j}\right)$ and $R\left(\varphi_{j}\right)$ are denoted as

$$
T\left(L_{j}\right)=\left[\begin{array}{ccccc}
1 & L_{j} & 0 & 0 & 0 \\
0 & 1 & 0 & 0 & 0 \\
0 & 0 & 1 & L_{j} & 0 \\
0 & 0 & 0 & 1 & 0 \\
0 & 0 & 0 & 0 & 1
\end{array}\right],
$$

$$
\begin{aligned}
& R\left(\varphi_{j}\right) \\
& \quad=\left[\begin{array}{ccccc}
\cos \left(\varphi_{j}\right) & 0 & \sin \left(\varphi_{j}\right) & 0 & 0 \\
0 & \cos \left(\varphi_{j}\right) & 0 & \sin \left(\varphi_{j}\right) & 0 \\
-\sin \left(\varphi_{j}\right) & 0 & \cos \left(\varphi_{j}\right) & 0 & 0 \\
0 & -\sin \left(\varphi_{j}\right) & 0 & \cos \left(\varphi_{j}\right) & 0 \\
0 & 0 & 0 & 0 & 1
\end{array}\right] .
\end{aligned}
$$

Therefore, the UMS transmission ray matrix can be represented by a general $5 \times 5$ transfer matrix as

$$
M=T\left(L_{n}\right) \cdot \prod_{i=1}^{i=N}\left\{R\left(\varphi_{i}\right) \cdot M_{i}\left(R, A_{i}\right) \cdot T\left(L_{i}\right)\right\} .
$$

The system transfer matrix $M$ exhibits beam properties of the bundle of the narrow beam along the chief ray of the UMS. The input ray matrix $r_{\text {in }}=\left[\begin{array}{lllll}0 & 0 & 0 & 0 & 1\end{array}\right]^{T}$; then the output ray matrix $r_{0}=M \cdot r_{\text {in }}=\left[\begin{array}{lllll}r_{o x} & r_{o x}^{\prime} & r_{o y} & r_{o y}^{\prime} & 1\end{array}\right]^{T}$.

To analyze the influence of all possible perturbations, the deviations of the output beam in position and direction should be our concerns. The relative amount of change in terms of the deviations of the output beam after the perturbations in position and direction can be calculated numerically from the term $r_{o}$.

We define the position deviation $L_{D o}=\sqrt{r_{o x}^{2}+r_{o y}^{2}}$ and direction deviations $L_{\theta x}=r_{o x}^{\prime}$ and $L_{\theta y}=r_{o y}^{\prime}$. The symbols $L_{D o}, L_{\theta x}$, and $L_{\theta y}$ are denoted as the output beam's position and orientation error referred to as the ideal output beam's orientation (no perturbations included).

Once given the configuration parameters of $\mathrm{BHWC}$, the calculation of each reflection's parameter $\left(A_{i}, L_{i}, \varphi_{i}\right)$ can be deduced from Snell's law in vector form.

The parameter set $\left(A_{j}, L_{j}, \varphi_{j}\right)$ are used to characterize the $j$ th reflection-transmission matrix, where $\varphi_{j}$ is the dihedral 


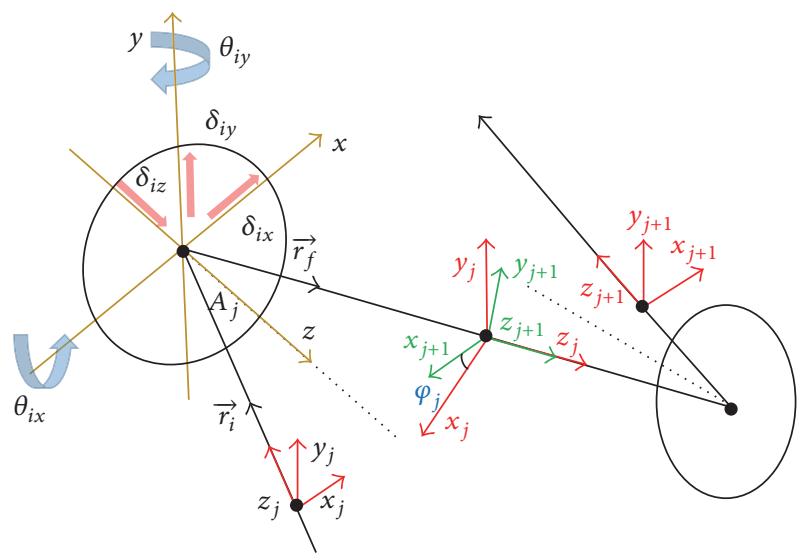

(a)

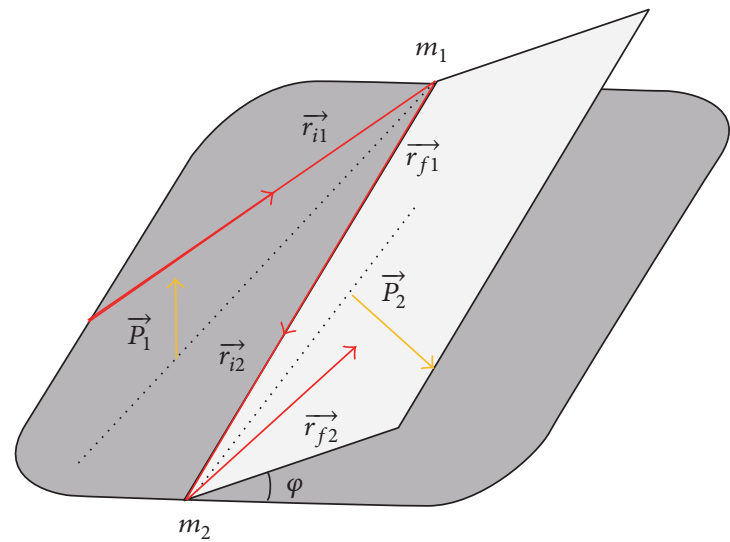

(b)

FIGURE 4: Rotation angle $\varphi$ for an off-axis reflection between two consecutive off-axis reflections onto the mirror $m_{1}$ and $m_{2}$. (a) Reflections and corresponding coordinate system for the UMS in the jth reflection. (b) Demonstration of the rotation angle between the two successive reflection planes.

angle between the two consecutive incident planes of the $j$ th and $(j+1)$ th reflection and $A_{j}$ is the incident angle and $L_{j}$ is the propagating distance of the chief ray between the two reflections. Figure 4(b) describes the method to figure out $\varphi_{j}$ based on the known incident ray vector $\overrightarrow{r_{i 1}}$ and reflection ray vector $\overrightarrow{r_{f 1}}$ calculated based on single ray tracing method. Thus, for example, $\overrightarrow{P_{1}}=\overrightarrow{r_{f 1}} \times \overrightarrow{r_{i 1}}$ and $\overrightarrow{P_{2}}=\overrightarrow{r_{f 2}} \times \overrightarrow{r_{i 2}}$, where $\overrightarrow{r_{i 2}}=\overrightarrow{r_{f 1}}$; we can obtain $\varphi_{j}$ using

$$
\varphi_{j}= \pm \cos ^{-1}\left(\frac{\left|\overrightarrow{P_{1}} \cdot \vec{P}_{2}\right|}{\left|\overrightarrow{P_{1}}\right| \cdot\left|\overrightarrow{P_{2}}\right|}\right) .
$$

The absolute value of $\varphi_{j}$ from (25) is always smaller than $90^{\circ}$. Considering that the coordinate system will change once between the left-handed system and right-handed system for each reflection, we define the initial coordinate system as lefthanded and $\varphi_{j}$ should be positive for the even number of reflection times for the UMS and negative for the odd number of reflection times for the UMS.

\section{Numerical Analysis Results}

According to the method presented above, the generalized spherical reflection ray matrix is applicable only when the input ray shooting on the spot is superimposed with the point on the perturbation or element axis. The feature of the UMS indicates that the reflecting point on the objective mirrors $M_{1}$ and $M_{2}$ is almost superimposed on the same point which is the center of the mirror's aperture while the reflecting points on the field mirror $F$ are distributed with two-row images pattern with defined column and row spacing. The misaligned reflection ray matrix (matrix in (20)) cannot be used for the analysis of the field mirror $F$. In essence, the UMS is a conjugated resonator in which all mirrors have specific relative relationship in 3D space. So the perturbation of the field mirror can be regarded as the perturbation of the objective mirrors; it is enough to present the optical axis sensitivity for the objective mirrors $M_{1}$ and $M_{2}$.

The analysis of axis's perturbation in this paper is based on the coplane condition between the reflection plane and the translational perturbation plane $\left(T_{i x}-O-T_{i z}\right.$ plane defined in Figure 5). However, the reflection plane (meridional plane, namely, $T_{i x}-O-T_{i z}$ plane) for each reflection in the UMS varied each time, and the $T_{i x}-O-T_{i z}$ plane is fixed as a precondition in the derivation based on Figure 5. So it is necessary to convert the perturbation vector $\left[\begin{array}{lllll}\delta_{i x} & \delta_{i y} & \theta_{i x} & \theta_{i y} & 1\end{array}\right]^{T}$ to the perturbation vector $\left[\begin{array}{lllll}\delta_{i x}^{j} & \delta_{i y}^{j} & \theta_{i x}^{j} & \theta_{i y}^{j} & 1\end{array}\right]^{T}$ in the reflection plane for $j$ th reflection by using the coordinate rotation matrix $R\left(\alpha_{j}\right)$ from (23); we modified the perturbation vector as

$$
\begin{aligned}
& {\left[\begin{array}{lllll}
\delta^{j}{ }_{i x} & \delta^{j}{ }_{i y} & \theta^{j}{ }_{i x} & \theta^{j}{ }_{i y} & 1
\end{array}\right]^{T}} \\
& \quad=R\left(\alpha_{j}\right) \cdot\left[\begin{array}{lllll}
\delta_{i x} & \delta_{i y} & \theta_{i x} & \theta_{i y} & 1
\end{array}\right]^{T} .
\end{aligned}
$$

The definitions of four dimensionless sensitivity factors SD1, SD2, ST1, and ST2 are given as $[14,19]$

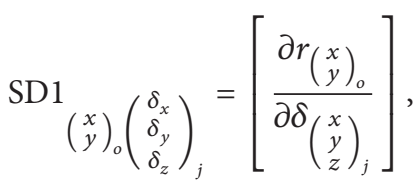

$$
\begin{aligned}
& \operatorname{SD2}\left(\begin{array}{l}
x \\
y
\end{array}\right)_{o}\left(\begin{array}{l}
\theta_{x} \\
\theta_{y}
\end{array}\right)_{j}=\frac{1}{R} \cdot\left[\frac{\partial r_{\left(\begin{array}{l}
x \\
y
\end{array}\right)_{o}}}{\partial \theta_{\left(\begin{array}{c}
x \\
y
\end{array}\right)_{j}}}\right] \text {, }
\end{aligned}
$$




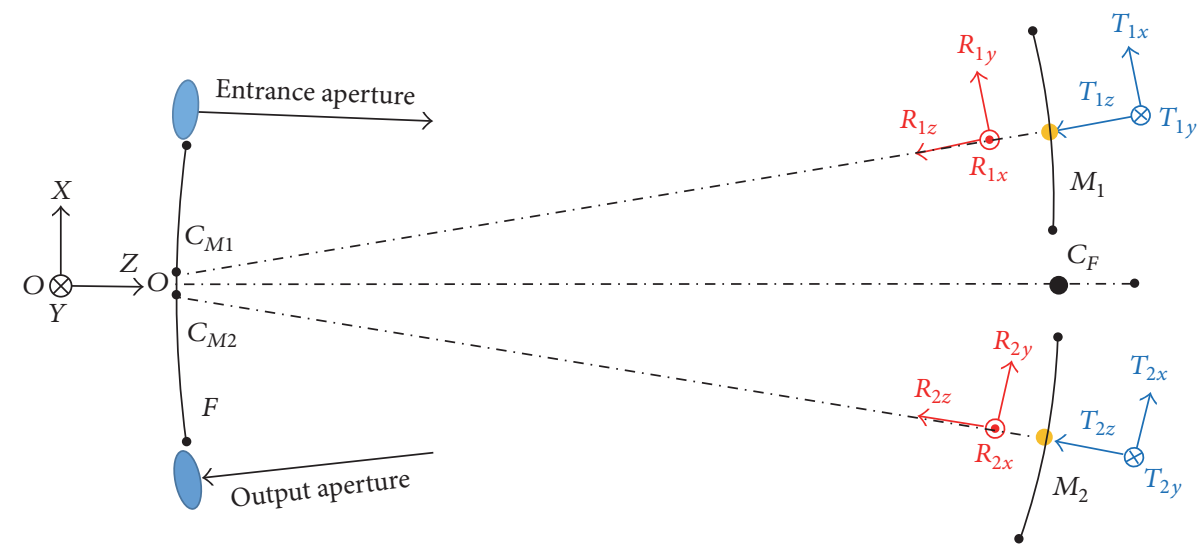

Figure 5: The definition of the objective mirror's translational and rotational perturbation axis $T_{i x}-T_{i y}-T_{i z}$ and $R_{i x}-R_{i y}-R_{i z}(i$ can be 1 or 2 indicating the mirrors $M_{1}$ and $M_{2}$, resp.). $X-Y-Z$ is the global coordinate system. $C_{F}, C_{M 1}$, and $C_{M 2}$ are the centers of curvature of the mirrors $F, M_{1}$, and $M_{2}$, respectively.

$$
\begin{aligned}
& \operatorname{ST1}\left(\begin{array}{l}
\theta_{x} \\
\theta_{y}
\end{array}\right)_{o}\left(\begin{array}{l}
\delta_{x} \\
\delta_{y} \\
\delta_{z}
\end{array}\right)_{j}=R \cdot\left[\frac{\partial r_{\left(\begin{array}{l}
x \\
y
\end{array}\right)_{o}}^{\prime}}{\partial \delta\left(\begin{array}{l}
x \\
y \\
z
\end{array}\right)_{j}}\right], \\
& \operatorname{ST2}\left(\begin{array}{l}
\theta_{x} \\
\theta_{y}
\end{array}\right)_{o}\left(\begin{array}{l}
\theta_{x} \\
\theta_{y}
\end{array}\right)_{j}=\left[\begin{array}{c}
\partial r^{\prime}\left(\begin{array}{l}
x \\
y
\end{array}\right)_{o} \\
\partial \theta_{\left(\begin{array}{l}
x \\
y
\end{array}\right)_{j}}
\end{array}\right] \text {, }
\end{aligned}
$$

where $r\left(\begin{array}{l}x \\ y\end{array}\right)_{0}$ and $\left.r_{(}^{\prime} \begin{array}{l}x \\ y\end{array}\right)_{0}$ are the induced $x$ or $y$ component of optical axis displacement and optical axis tilt (in radians) on the output aperture as the result of $j$ th mirror's axial decentration along the translational axis $T_{i x}, T_{i y}$, or $T_{i z}$ with an amount of $\delta\left(\begin{array}{l}x \\ y \\ z\end{array}\right)_{j}$ or angular misalignment around rotating axis $R_{i x}$ or $R_{i y}$ with an amount $\theta_{\left(\begin{array}{l}x \\ y\end{array}\right)_{j}}$ (in radians).

The sensitivity of optical axis perturbation can be obtained by solving (27) One thing to note is that the incident angle for each reflection should be smaller than $5^{\circ}$ in a defined BHWC when choosing the initial parameters $\left(n, R, d_{r}, d_{c}\right)$; if not, the ray matrix method will result in large errors.

In our numerical simulations, we choose the given parameters $\left(R, n, d_{c}\right)$ and vary $d_{r}$ to establish different UMS configurations. We observe the relationship between sensitivity factors $\mathrm{SD}_{1}, \mathrm{SD}_{2}, \mathrm{ST}_{1}$, and $\mathrm{ST}_{2}$ with the ratio of $d_{r} / d_{c}$.

In BHWC, we have fixed parameters $R=600 \mathrm{~mm}, n=$ 5 , and $d_{c}=15 \mathrm{~mm}$ and varied $d_{r} / d_{c}$ ranging from 0 to 3 ; we calculate the sensitivity factors of the objective mirrors $M_{1}$ and $M_{2}$ with possible independent perturbation sources $\left(\delta_{j x}, \delta_{j y}, \delta_{j z}, \theta_{i x}, \theta_{i y}\right)$ with very small value.

It can be seen from the simulation results (Figures 6-9) that the movement of optical axis of the objective mirrors demonstrates some characteristics:
(1) The sensitivity factor of the system is not dependent on the choice of $d_{r} / d_{c}$ but is dependent on the choice of parameter $n$, that is, the reflection times $N=4 n$ (BHWC) or $N=4 n-2$ (PBWC). The larger the reflection times, the larger the value of the sensitivity factor, which means the lower tolerance of the objective mirror's alignment is required.

(2) In terms of SD, the axial misalignments along the translational axes $T_{i x}$ and $T_{i y}$ for the objective mirrors $M_{1}$ or $M_{2}$ have the same effects on the position error of the output beam, which means $\mathrm{SD} 1_{\text {roxi }}=$ SD $1_{\text {royi }}(i=1,2)$, and the angular misalignment around $R_{i x}$ has similar effects on the output beam's position when compared with the axial misalignment along $T_{i x}$ or $T_{i y}$, which means $\mathrm{SD} 2_{r o \theta_{x} i}=\mathrm{SD} 1_{\text {roxi }}(i=$ $1,2)$. It can be summarized as

$$
\mathrm{SD} 1_{\text {roxi }}=\mathrm{SD} 1_{\text {royi }}=\mathrm{SD} 2_{\text {ro } \theta_{x} i}
$$

(3) regarding ST, we have ten lines to represent the sensitivity factor in direction error; there are four couple lines showing the same relationships with the term $d_{r} / d_{c}$; they can be summarized as

$$
\begin{aligned}
& \mathrm{ST} 1_{\theta_{x} o x i}=\mathrm{ST} 2_{\theta_{x} o \theta_{x}}, \\
& \mathrm{ST} 1_{\theta_{y} o y i}=\mathrm{ST} 2_{\theta_{y} o \theta_{y} i}, \\
& \mathrm{ST} 1_{\theta_{y} o x i}=\mathrm{ST} 2_{\theta_{x} o \theta_{y} i}, \\
& \mathrm{ST} 1_{\theta_{x} o y i}=\mathrm{ST} 2_{\theta_{y} o \theta_{x} i},
\end{aligned}
$$

where $i=1,2$ representing the objective mirrors $M_{1}$ and $M_{2}$.

(4) The sensitivity factors of $M_{1}$ and $M_{2}$ manifest the feature of symmetry regarding $\mathrm{ST} 1_{\theta_{x} \text { oxi }}$ and $\mathrm{ST} 1_{\theta_{y} \text { oxi }}$, 

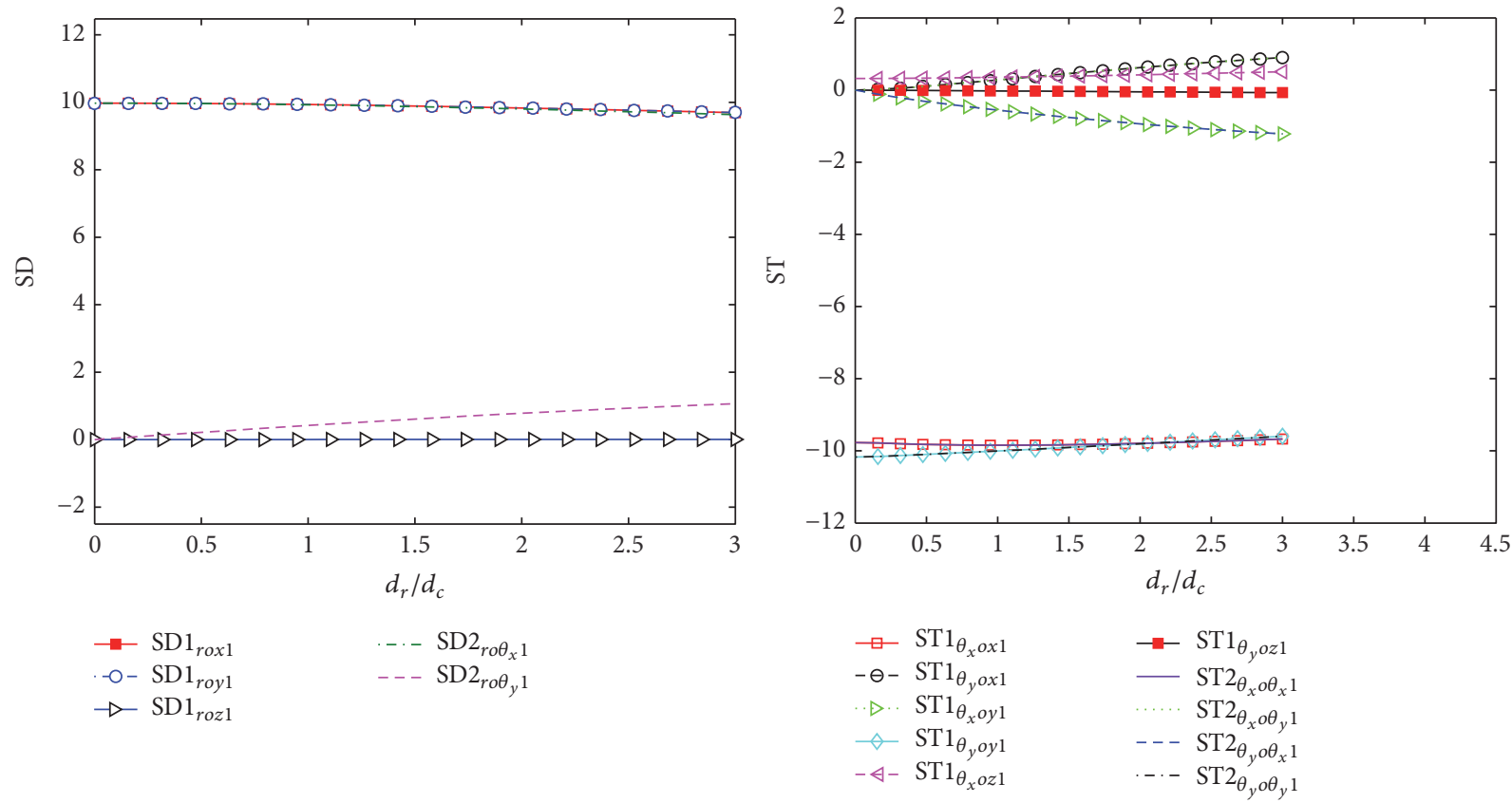

FIGURE 6: Sensitivity factors SD1, SD2, ST1, and ST2 characterizing the movement of the optical axis on the mirror $M_{1}$ with fixed column spacing $d_{c}=15 \mathrm{~mm}, R=600 \mathrm{~mm}$, and $n=5$. We change the ratio of $d_{r} / d_{c}$ between 0 and 3 to get different configurations of BHWC.
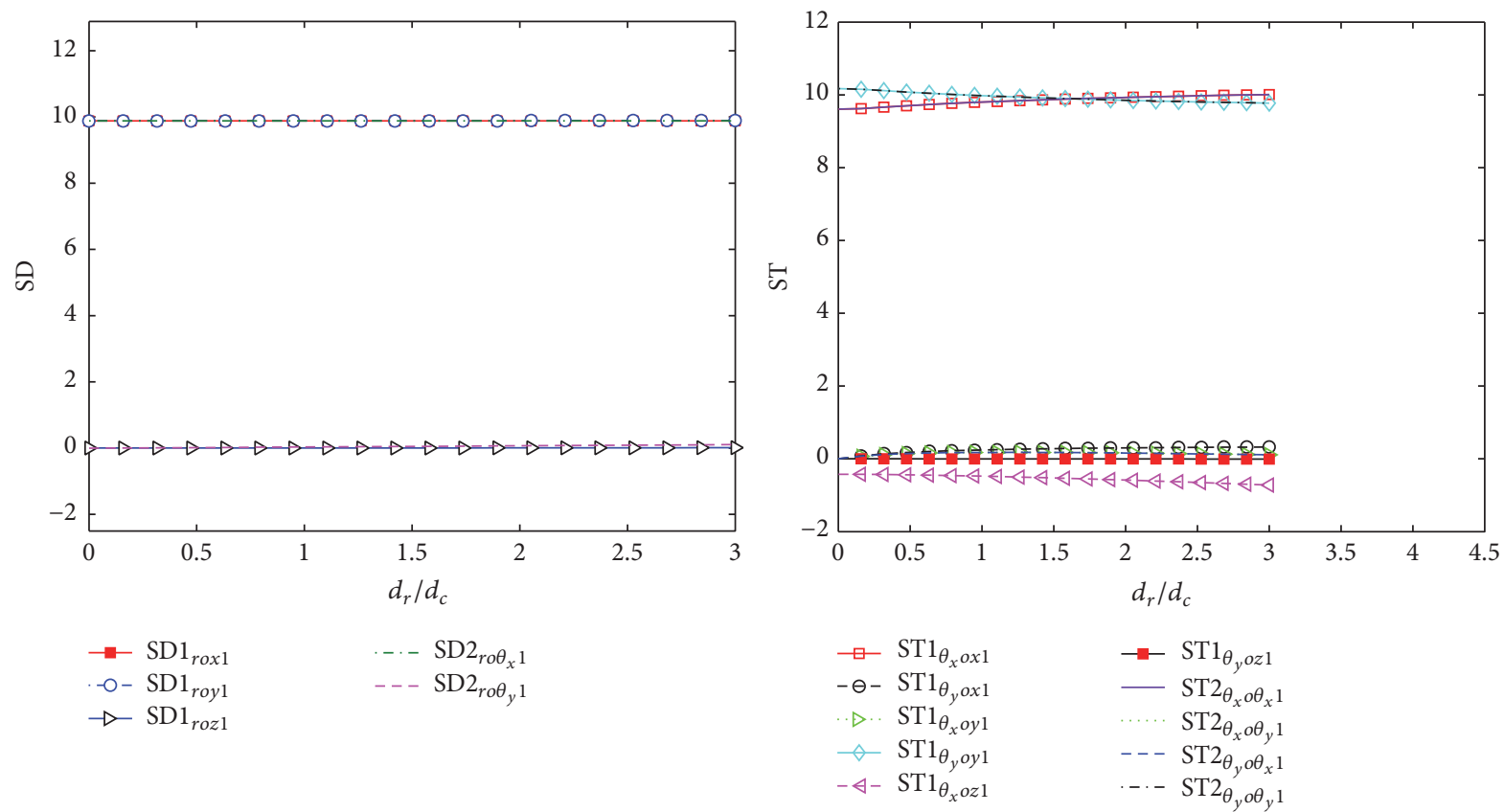

FIGURE 7: Sensitivity factors SD1, SD2, ST1, and ST2 characterizing the movement of the optical axis on the mirror $M_{2}$ with fixed column spacing $d_{c}=15 \mathrm{~mm}, R=600 \mathrm{~mm}$, and $n=5$. We change the ratio of $d_{r} / d_{c}$ between 0 and 3 to get different configurations of BHWC.

which can be explained by the symmetry position of the BHWC's objective mirrors.

(5) All possible perturbations $\left(\delta_{j x}, \delta_{j y}, \delta_{j z}, \theta_{i x}, \theta_{i y}\right)$ influence the direction and the position of the output images. However, they have different weights on the influence of the optical axis's variations related to the ideal output orientation. Considering the factor SD, the influence from $\left(\delta_{j x}, \delta_{j y}, \theta_{i x}\right)$ plays an important role in changing the position of the output beam when compared with terms $\left(\delta_{j z}, \theta_{j y}\right)$. Moreover, considering ST, the angular misalignment in $x$ direction 

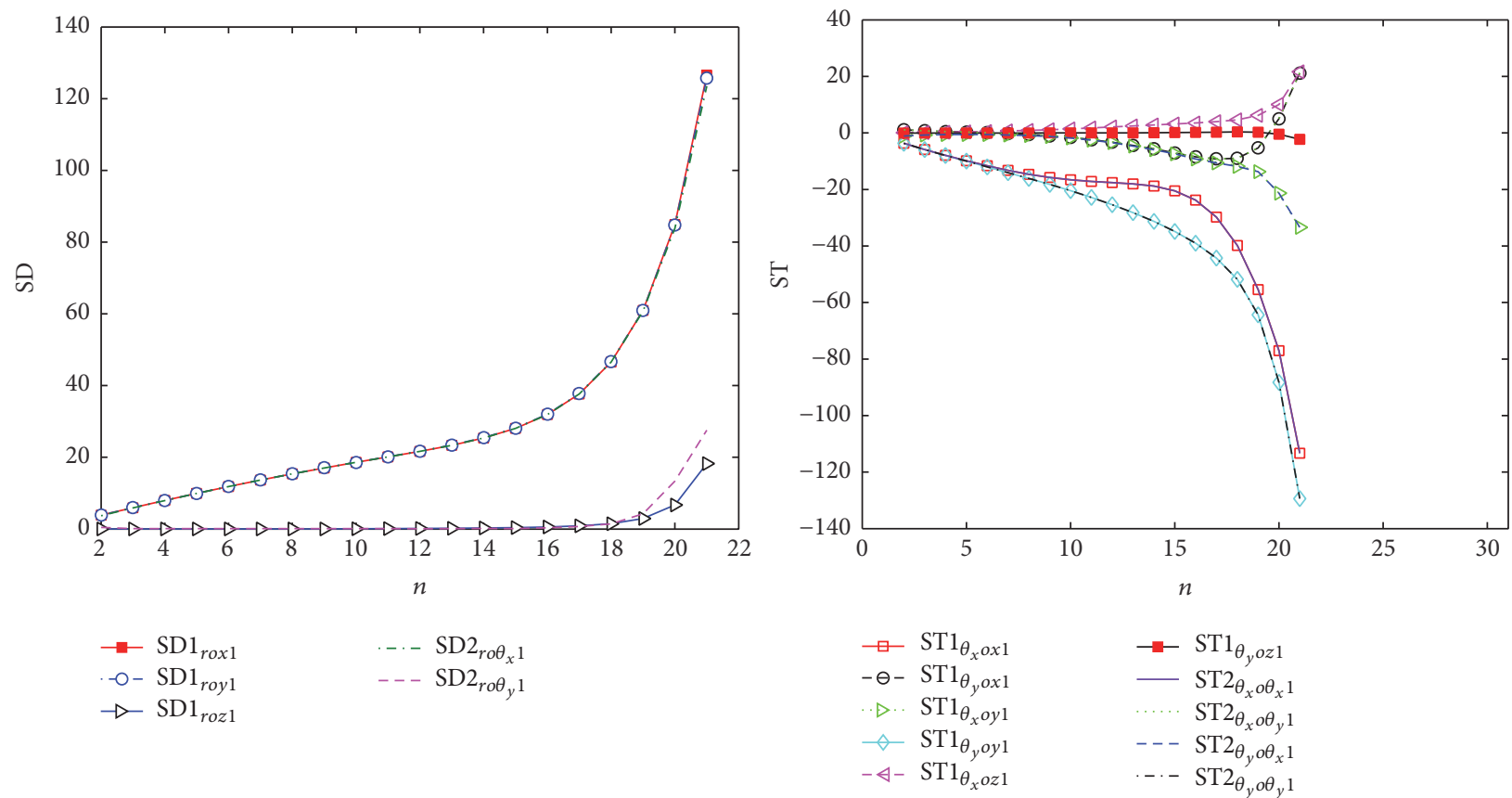

FIGURE 8: Sensitivity factors SD1, SD2, ST1, and ST2 considering the possible perturbation of the optical axis on the mirror $M_{1}$ with parameters $R=600 \mathrm{~mm}$. The parameter $n$ varies ranging from 2 to 21 to get different configurations of BHWC.
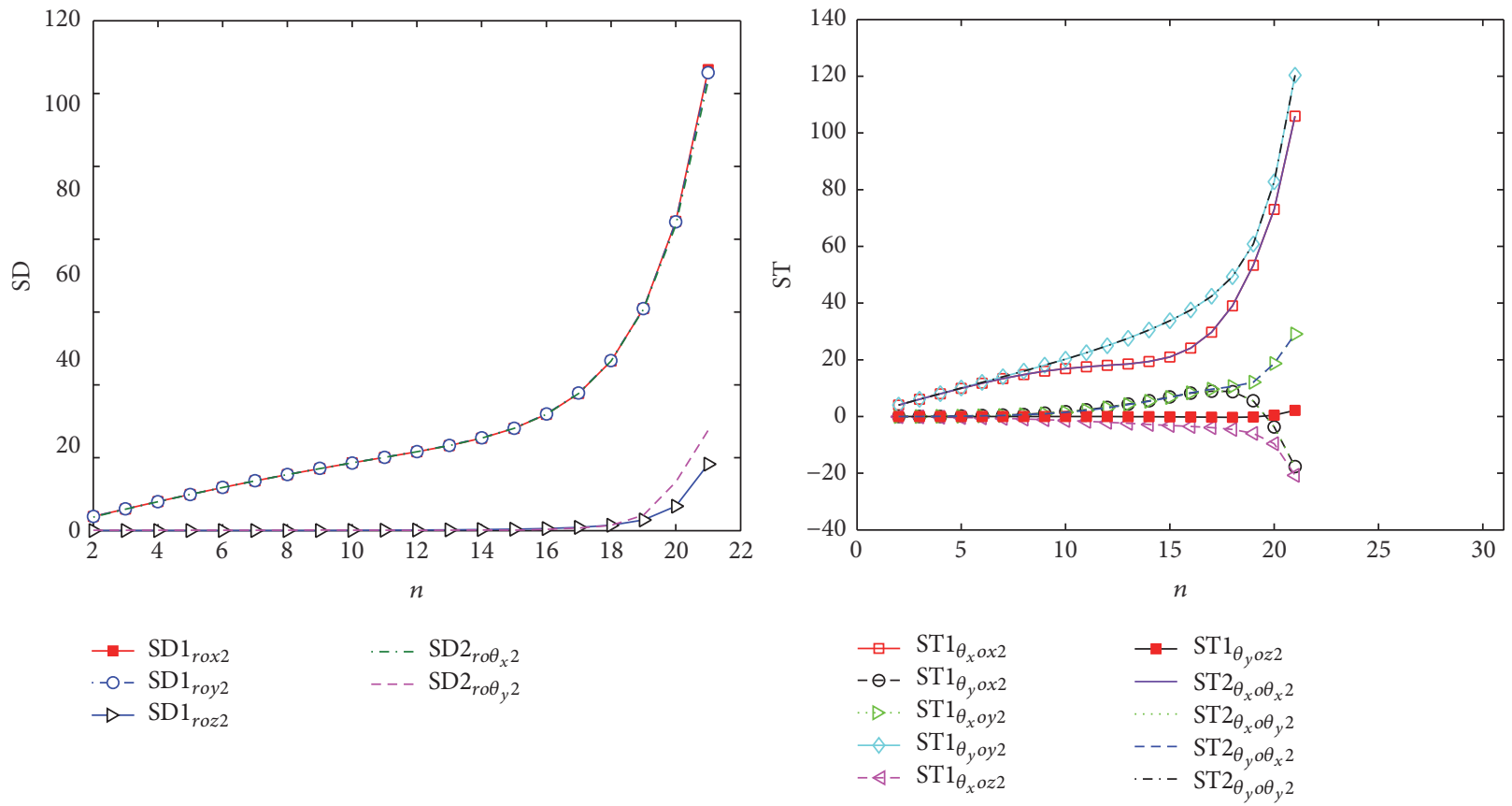

FIGURE 9: Sensitivity factors SD1, SD2, ST1, and ST2 considering the possible perturbation of the optical axis on the mirror $M_{2}$ with parameters $d_{c}=d_{r}=15 \mathrm{~mm}$ and $R=600 \mathrm{~mm}$. The parameter $n$ changes ranging from 2 to 21 to get different configurations of BHWC.

is mainly influenced by element's axial misalignment along $T_{i x}$ and angular misalignment rotating around $R_{i x}$. The angular misalignment in $y$ direction is mainly influenced by element's axial misalignment along $T_{i y}$ and angular misalignment rotating around $R_{i y}$.

\section{Conclusion}

In summary, the generalized ray matrix for the spherical mirror reflection has been derived in a new view, which is based on the general model of a paraxial misaligned element. The spherical mirror transfer matrix considers all possible 
perturbation sources, two kinds of angular misalignments, $\theta_{i x}$ and $\theta_{i y}$, and three kinds of axial displacements, $\delta_{i x}, \delta_{i y}$, and $\delta_{i z}$. BHWCs have been chosen as examples to present the optical axis alignment properties of the multipass cell for the first time. The sensitivity factors SD1, SD2, ST1, and ST2 are calculated numerically when BHWCs are in different configurations. The main factors that result in the deviation of the output beam's position and direction have been found for the first time. Regarding the position error of the output beam, the axial displacement along $T_{i x}$ and $T_{i y}$ axes and the angular rotation around $R_{i x}$ of the objective mirror have more weights than other kinds of perturbation sources. Regarding misalignment amount of the direction of the output beam, the misaligned slope deviation about $x$-axis is mainly influenced by the axial displacement $\delta_{i x}$ and angular rotation $\theta_{i x}$ of the objective mirror. The misaligned slope deviation about $y$ axis is mainly influenced by the axial displacement error $\delta_{i y}$ and angular rotation error $\theta_{i y}$ of the objective mirror. The increase in the number of the reflection times will decrease the stability of the system and make the system more sensitive to the perturbations. The misalignment of $T_{i z}$ axis $\left(\delta_{i z}\right)$ has the least contributions to the output beam. The choice of the column spacing $d_{c}$ or the row spacing $d_{r}$ does not have relations to the optical axis sensitivity of the objective mirrors. The method can also be applied to the analysis of various kinds of multipass cells, such as PBWC, three-objective/fourobjective multipass matrix system, and Herriott cell. These results are important for the multipass cell's design and long-term stability control for the high accuracy alignment improvement for the multipass gas cell's construction.

\section{Competing Interests}

There is no conflict of interests regarding the publication of this manuscript.

\section{Acknowledgments}

This research was funded by Tsinghua University and National Major Scientific Instruments and Device Development Project of China (Grant no. 2012YQ200182).

\section{References}

[1] K. Chen, H. Yang, L. Sun, and G. Jin, "Generalized method for calculating astigmatism of the unit-magnification multipass system," Applied Optics, vol. 49, no. 10, pp. 1964-1971, 2010.

[2] Y. J. Kim and U. Platt, Advanced Environmental Monitoring, chapter 1, 2007.

[3] J. U. White, "Long optical paths of large aperture," Journal of the Optical Society of America, vol. 32, no. 5, p. 285, 1942.

[4] D. Herriott, H. Kogelnik, and R. Kompfner, "Off-axis paths in spherical mirror interferometers," Applied Optics, vol. 3, no. 4, pp. 523-526, 1964.

[5] B. Tuzson, M. Mangold, H. Looser, A. Manninen, and L. Emmenegger, "Compact multipass optical cell for laser spectroscopy,” Optics Letters, vol. 38, no. 3, pp. 257-259, 2013.
[6] W. H. Kohn, "Astigmatism and white cells: theoretical considerations on the construction of an anastigmatic white cell," Applied Optics, vol. 31, no. 31, pp. 6757-6764, 1992.

[7] H. J. Bernstein and G. Herzberg, "Rotation-Vibration spectra of diatomic and simple polyatomic molecules with long absorbing paths. I. The spectrum of fluoroform $\left(\mathrm{CHF}_{3}\right)$ from $2.4 \mu$ to $0.7 \mu$," The Journal of Chemical Physics, vol. 16, no. 1, pp. 30-39, 1948.

[8] T. H. Edwards, "Multiple-traverse absorption cell design," Journal of the Optical Society of America, vol. 51, no. 1, pp. 98-102, 1961.

[9] H. M. Pickett, G. M. Bradley, and H. L. Strauss, "A new white type multiple pass absorption cell," Applied Optics, vol. 9, no. 10, pp. 2397-2398, 1970.

[10] S. M. Chernin, "Development of optical multipass matrix systems," Journal of Modern Optics, vol. 48, no. 4, pp. 619-632, 2001.

[11] S. M. Chernin, "Promising version of the three-objective multipass matrix system," Optics Express, vol. 10, no. 2, pp. 104-107, 2002.

[12] S.-C. Sheng, "Optical-axis perturbation singularity in an out-ofplane ring resonator," Optics Letters, vol. 19, no. 10, pp. 683-685, 1994.

[13] M. Chen, J. Yuan, X. Long, Z. Kang, Z. Wang, and Y. Li, "General beam position controlling method for 3D optical systems based on the method of solving ray matrix equations," Optics \& Laser Technology, vol. 54, pp. 343-346, 2013.

[14] J. Yuan and X. Long, "Optical-axis perturbation in nonplanar ring resonators," Optics Communications, vol. 281, no. 5, pp. 1204-1210, 2008.

[15] J. Yuan, M. Chen, Y. Li, Z. Tan, and Z. Wang, "Reanalysis of generalized sensitivity factors for optical-axis perturbation in nonplanar ring resonators," Optics Express, vol. 21, no. 2, pp. 2297-2306, 2013.

[16] J. Yuan, X. Long, and M. Chen, "Generalized ray matrix for spherical mirror reflection and its application in square ring resonators and monolithic triaxial ring resonators," Optics Express, vol. 19, no. 7, pp. 6762-6776, 2011.

[17] A. E. Siegman, "Ray optics and ray matrices," in Lasers, chapter 15, pp. 581-609, University Science Books, 1986.

[18] X. Liu and K.-H. Brenner, "Minimal optical decomposition of ray transfer matrices," Applied Optics, vol. 47, no. 22, pp. E88E98, 2008.

[19] D. Wen, D. Li, and J. Zhao, "Generalized sensitivity factors for optical-axis perturbation in nonplanar ring resonators," Optics Express, vol. 19, no. 20, pp. 19752-19757, 2011. 


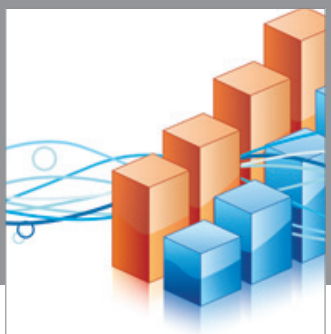

Advances in

Operations Research

vatem alat4

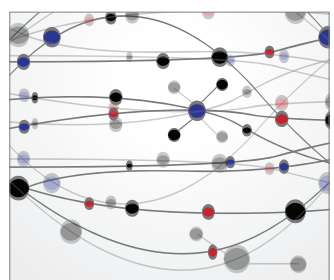

\section{The Scientific} World Journal
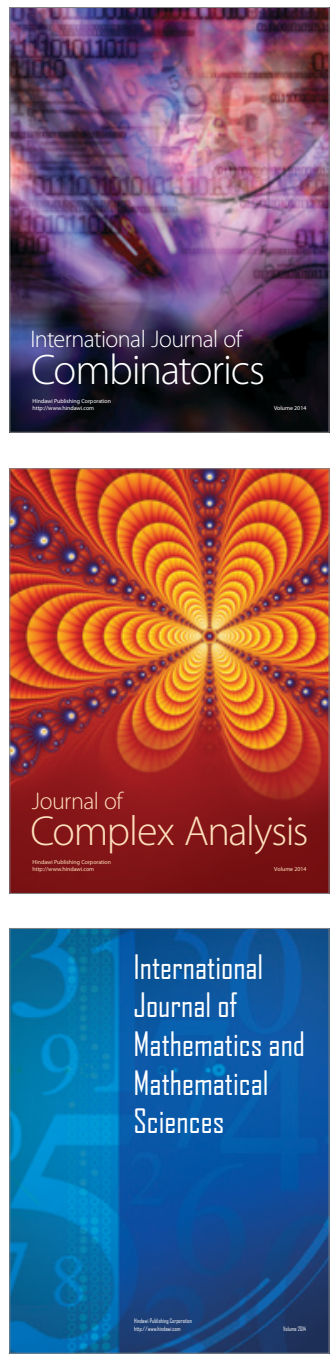
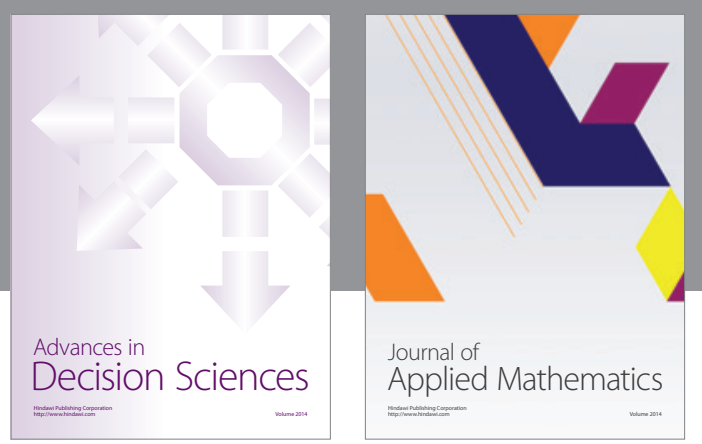

Algebra

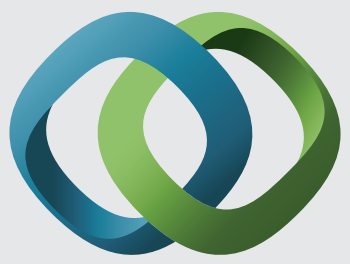

\section{Hindawi}

Submit your manuscripts at

https://www.hindawi.com
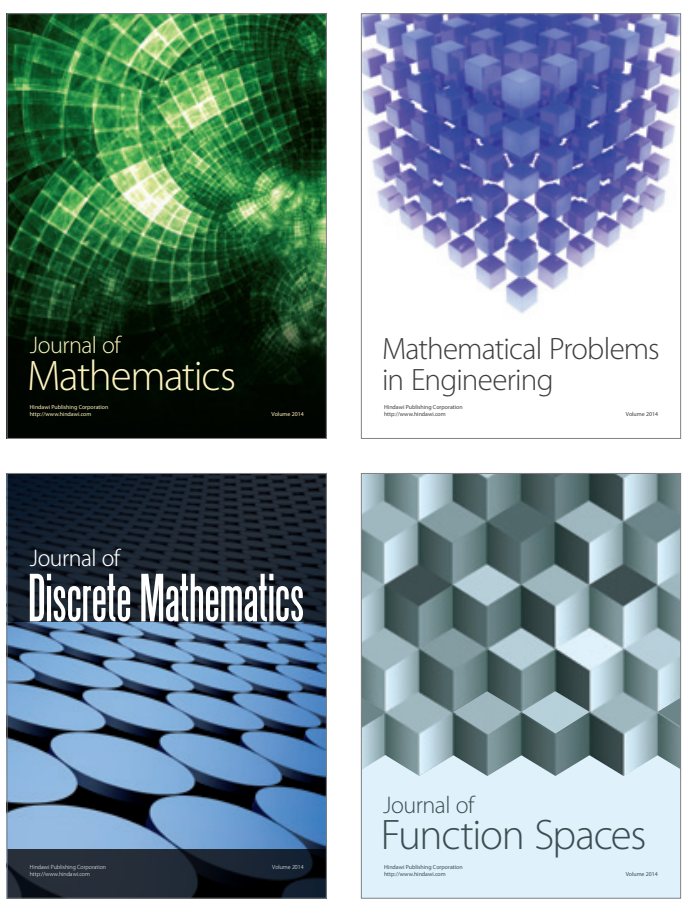

Mathematical Problems in Engineering
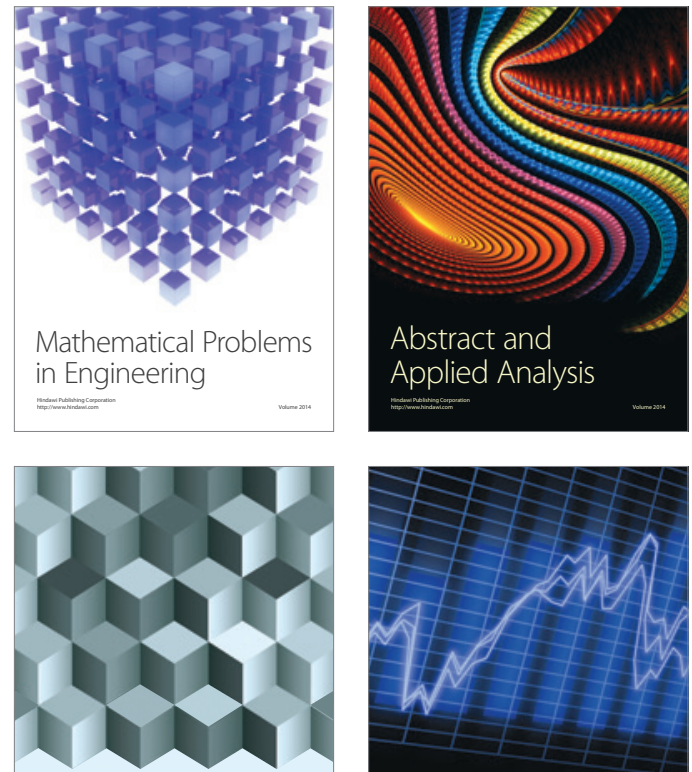

Journal of

Function Spaces

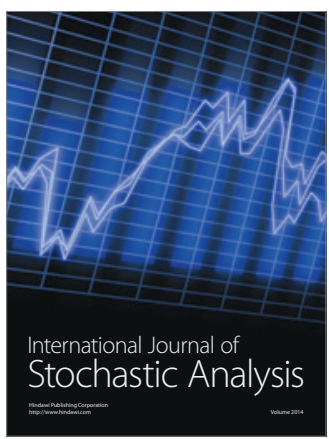

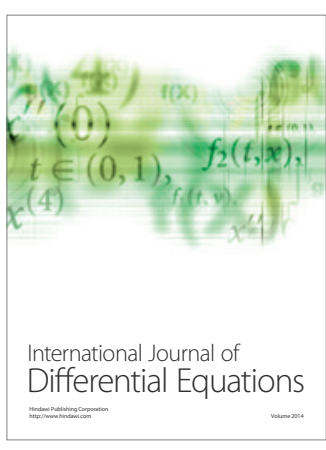
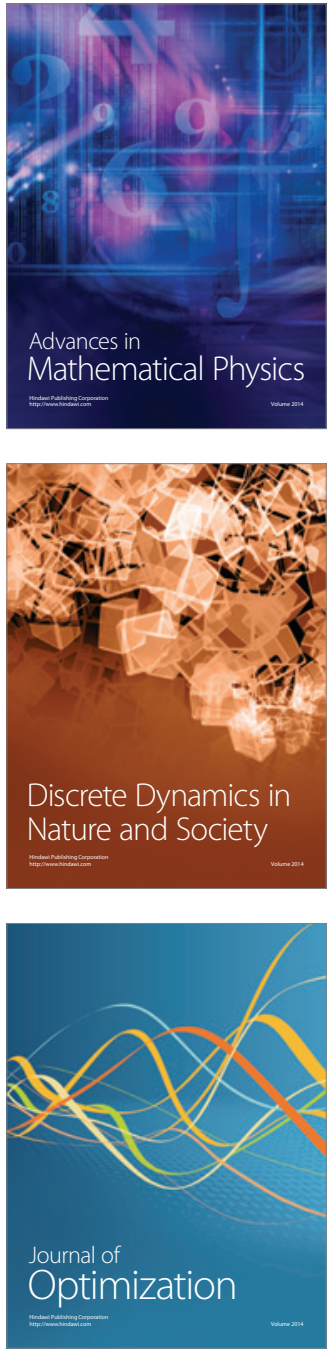